\title{
PAX7 expression in sarcomas bearing the EWSR1-NFATC2 translocation
}

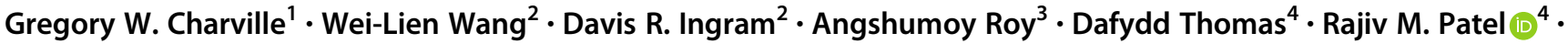 \\ Jason L. Hornick ${ }^{5}$ Matt van de Rijn ${ }^{1}$ - Alexander J. Lazar $\mathbb{D}^{2}$
}

Received: 1 May 2018 / Accepted: 5 May 2018 / Published online: 9 July 2018

(c) United States \& Canadian Academy of Pathology 2018

We thank Dr. Grunewald and colleagues for reading our manuscript [1], and we are happy to respond to their comments. In their letter, Grunewald et al. [2] raise the question of whether EWSR1-NFATC2-positive sarcomas really are Ewing sarcomas. They provide information from their interesting publication that appeared in Oncotarget to support the idea that EWSR1-NFATC2-positive sarcomas are transcriptionally distinct from tumors with EWSRI-FLII translocation. Unfortunately, their manuscript was published after the publication of our paper, and therefore we could not reference their data in our report. They do mention that we did not "take into account more recent reports in the literature that EWSR1-NFATC2-positive sarcomas may constitute an own entity." Here, their letter cites a single article summarizing a meeting of the European Interdisciplinary Ewing Sarcoma Research Summit [3], published in 2016. Grunewald et al. feel that we have overlooked this publication, which would have served, in their view, as a guide to the appropriate classification of the EWSR1-NFATC2 variant tumors. However, the cited publication, albeit a generally helpful and important commentary, which references unpublished gene expression studies

$\triangle$ Gregory W. Charville

gwc@stanford.edu

1 Department of Pathology, Stanford University School of Medicine, Stanford, CA, USA

2 Department of Pathology, The University of Texas MD Anderson Cancer Center, Houston, TX, USA

3 Departments of Pathology \& Immunology and Pediatrics, Texas Children's Hospital, Baylor College of Medicine, Houston, TX, USA

4 Department of Pathology, University of Michigan School of Medicine, Ann Arbor, MI, USA

5 Department of Pathology, Brigham and Women's Hospital, Boston, MA, USA of EWSR1-NFATC2-translocated tumors, presents no primary data to distinguish these as a unique entity. Also, this publication does not provide specific recommendations to guide classification of the EWSR1-NFATC2 variant.

In our decision to analyze EWSRI-NFATC2 cases as members of the "Ewing family," we followed in the footsteps of several preceding publications that treat EWSRINFATC2 tumors as Ewing sarcoma variants [4-17]. Notably, the College of American Pathologists' [26] Protocol for the Examination of Specimens from Patients with Soft Tissue Tumors version 4.0.0.0 (published in June 2017) also lists the EWSR1-NFATC2 fusion as a "molecular event" in "Ewing sarcoma." For its part, the World Health Organization's [27] Classification of Tumours of Soft Tissue and Bone (2013) states in reference to EWSRI-NFATC2 tumors that "whether these cases should be defined as Ewing sarcoma, or represent separate tumor types is unknown, but currently they are treated in a similar fashion."

We too feel that the question of whether the EWSRINFATC2 tumors should be treated as an entity completely distinct from EWSRI-ETS tumors is not yet resolved. However, knowing that the underlying translocation could be relevant to these tumors' eventual classification, we were careful to indicate throughout our manuscript when our analyses involved specific "variants." Regardless of the nomenclature, the EWSRI-NFATC2 tumors, like the EWSRI-FLII and EWSRI-ERG cases, express PAX7, while lesions with a CIC-DUX4 translocation do not. This was the message of our manuscript. Importantly, a recent RNAsequencing study of small round-cell sarcomas [18], including gene expression data from 184 tumors, confirmed our observation that $P A X 7$ is one of the most differentially expressed genes in Ewing sarcoma relative to other small round -cell sarcomas.

We concur with Grunewald et al. that pathologists need to be cognizant of the limitations and specificity of PAX7 expression analyses. In fact, our paper references and 
discusses our prior study in which we found PAX7 to be expressed in both rhabdomyosarcoma and synovial sarcoma [19]. Therefore, like many antibodies employed in surgical pathology, we believe PAX7 would be best used in a panel of markers and in the appropriate clinicopathologic context. Although we did not make any assertions about the sensitivity of PAX7 relative to CD99, we did state that CD99 expression is "highly sensitive" for the identification of Ewing sarcoma and would indeed think it ill-advised to suggest that PAX7, or any marker for that matter, should wholly supplant a "tried and true" diagnostic tool such as CD99. Nevertheless, in our series, all molecularly proven Ewing sarcomas expressed PAX7 and, therefore, we think PAX7 expression may be a useful ancillary diagnostic tool for these tumors.

Grunewald et al. mention the importance of confirming the presence of an EWSRI translocation in tumor samples. We also think this is important-in both the "Materials and methods" and the "Results" section of our paper, we describe the molecular characterization of all PAX7negative cases in our initial cohort study using nextgeneration sequencing (NGS) of libraries prepared with an anchored multiplex PCR-based methodology. The vast majority of remaining PAX7-positive samples had been confirmed through routine clinical testing by FISH, RT$\mathrm{PCR}$, or sequencing methodologies, as has been the practice in our institutions for many years.

Grunewald et al. additionally inquire with regard to our characterization of a candidate EWSR1-FLI1-regulated $P A X 7$ enhancer. We focused on a single candidate regulatory element because it met the following criteria of an EWSR1-FLI1-regulated active enhancer: (1) the site is bound by EWSR1-FLI1 in SKNMC and A673 cell lines, (2) the site coincides with a peak of H3K27 acetylation in SKNMC and A673 cells that is lost with knockdown of the oncogenic fusion protein, (3) the site overlaps with a peak of H3K27 acetylation, which is gained in mesenchymal stem cells upon transgenic expression of the oncogenic fusion protein, and (4) the site coincides with a peak of H3K27 acetylation in three primary Ewing sarcoma specimens [20]. Other candidate EWSR1-FLI1 binding sites proximal to $P A X 7$, in our opinion, do not meet these stringent criteria. Still, given the complexity of distal regulatory elements in the human genome, we would not expect, nor did we suggest, that the element highlighted by our analysis represents the sole mechanism of PAX7 expression in Ewing sarcoma.

Lastly, Grunewald and colleagues point to the polymorphic nature of GGAA-microsatellites as a reason to doubt that such repeats would mediate "uniformly high" PAX7 expression in Ewing sarcoma. It is well established that EWSR1-FLI1 creates de novo enhancers at GGAA microsatellites to increase transcription of target genes
[20-23]. The EWSR1-FLI1-bound regulatory element that was highlighted in our study is centered on a microsatellite containing 18 GGAA motifs in the reference genome (hg19). These 18 consecutive motifs meet the established threshold of >10AA repeats required for "strong" reporter gene activity in studies of EWSR1-FLI1 transcriptional regulation and fall just within the range of 18-26 motifs representing the "optimal" length for EWSR1-FLI1-binding $[21,24,25]$. Moreover, analysis of the NROB1 promoter GGAA-microsatellite has shown that (1) stability of GGAA microsatellites during oncogenesis limits polymorphism within individual tumors and (2) "selection bias" restricts the case-to-case variability of GGAA-microsatellite length in Ewing sarcoma [24]. These findings predict robust gene expression mediated by a GGAA microsatellite like the one identified in our study.

In this age of precision medicine, topics such as the relationship of EWSR1-NFATC2 translocated Ewing or "Ewing-like" sarcomas to the canonical EWSRI-FLII translocated Ewing sarcomas are of critical importance, and we thank Grunewald et al. for reminding us of this. At this point, we believe that the jury is still out regarding this distinction and we look forward to the related discussions at the upcoming working group conference of the WHO Classification of Tumours of Soft Tissue and Bone. Establishing thresholds for designating an entity as truly distinct from another is a complex decision involving molecular data, clinical experience and practice, and, to a certain degree, social constructions. Although the designation of EWSR1-NFATC2 tumors is currently unresolved in our opinion, data such as those presented by Grunewald et al. and our own work, as well as the work of others around the globe, will hopefully bring additional clarity over time.

\section{Compliance with ethical standards}

Conflict of interest The authors declare that they have no conflict of interest.

\section{References}

1. Charville GW, Wang W-L, Ingram DR, et al. EWSR1 fusion proteins mediate PAX7 expression in Ewing sarcoma. Mod Pathol. 2017;30:1312-20.

2. Baldauf MC, Gerke JS, Orth MF, Dallmayer M, Baumhoer D, de Alava E, et al. Are EWSR1-NFATc2-positive sarcomas really Ewing sarcomas? Mod Pathol. 2018;31:997-99.

3. Kovar H, Amatruda J, Brunet E, et al. The second European interdisciplinary Ewing sarcoma research summit--A joint effort to deconstructing the multiple layers of a complex disease. Oncotarget. 2016;7:8613-24.

4. Petrovic M, Zhao B, Thangam M, et al. Ewing Sarcoma in the Right Ventricle. Tex Heart Inst J. 2016;43:458-60. 
5. Owosho AA, Ko E, Rosenberg HI, et al. Primary Ewing Family of Tumors of the Jaw has a better Prognosis compared to Tumors of Extragnathic sites. J Oral Maxillofac Surg. 2016;74:973-81.

6. Xiao W, Mohseny AB, Hogendoorn PCW, et al. Mesenchymal stem cell transformation and sarcoma genesis. Clin Sarcoma Res. 2013;3:10.

7. Parker BC, Zhang W. Fusion genes in solid tumors: an emerging target for cancer diagnosis and treatment. Chin $\mathrm{J}$ Cancer. 2013;32:594-603.

8. Cantile M, Marra L, Franco R, et al. Molecular detection and targeting of EWSR1 fusion transcripts in soft tissue tumors. Med Oncol. 2013;30:412.

9. Berghuis D, Schilham MW, Santos SJ, et al. The CXCR4CXCL12 axis in Ewing sarcoma: promotion of tumor growth rather than metastatic disease. Clin Sarcoma Res. 2012;2:24.

10. Berghuis D, Schilham MW, Vos HI, et al. Histone deacetylase inhibitors enhance expression of NKG2D ligands in Ewing sarcoma and sensitize for natural killer cell-mediated cytolysis. Clin Sarcoma Res. 2012;2:8.

11. Pan M-G, Xiong Y, Chen F. NFAT gene family in inflammation and cancer. Curr Mol Med. 2013;13:543-54.

12. van Maldegem AM, Bhosale A, Gelderblom HJ, et al. Comprehensive analysis of published phase I/II clinical trials between 1990-2010 in osteosarcoma and Ewing sarcoma confirms limited outcomes and need for translational investment. Clin Sarcoma Res. 2012;2:5.

13. Blau O. Molecular investigation of Ewing sarcoma: about detecting translocations. EMBO Mol Med. 2012;4:449-50.

14. Baumgart S, Glesel E, Singh G, et al. Restricted heterochromatin formation links NFATc2 repressor activity with growth promotion in pancreatic cancer. Gastroenterology. 2012;142:388-98. e1-7

15. Singh SK, Baumgart S, Singh G, et al. Disruption of a Nuclear NFATc2 Protein Stabilization Loop Confers Breast and Pancreatic Cancer Growth Suppression by Zoledronic Acid. J Biol Chem. 2011;286:28761-71.

16. Gamberi G, Cocchi S, Benini S, et al. Molecular Diagnosis in Ewing Family Tumors. J Mol Diagn JMD. 2011;13:313-24.

17. Szuhai K, Ijszenga $M$, de Jong $D$, et al. The NFATc2 gene is involved in a novel cloned translocation in a Ewing sarcoma variant that couples its function in immunology to oncology. Clin Cancer Res J Am Assoc Cancer Res. 2009; 15:2259-68.

18. Watson S, Perrin V, Guillemot D, et al. Transcriptomic definition of molecular subgroups of small round cell sarcomas. J Pathol. 2018;245:29-40.

19. Charville GW, Varma S, Forgó E, et al. PAX7 Expression in Rhabdomyosarcoma, Related Soft Tissue Tumors, and Small Round Blue Cell Neoplasms. Am J Surg Pathol. 2016;40:1305-15.

20. Riggi N, Knoechel B, Gillespie SM, et al. EWS-FLI1 Utilizes Divergent Chromatin Remodeling Mechanisms to Directly Activate or Repress Enhancer Elements in Ewing Sarcoma. Cancer Cell. 2014;26:668-81.

21. Guillon N, Tirode F, Boeva V, et al. The oncogenic EWS-FLI1 protein binds in vivo GGAA microsatellite sequences with potential transcriptional activation function. PLoS ONE. 2009;4:e4932.

22. Gangwal K, Sankar S, Hollenhorst PC, et al. Microsatellites as EWS/FLI response elements in Ewing's sarcoma. Proc Natl Acad Sci USA. 2008;105:10149-54.

23. García-Aragoncillo E, Carrillo J, Lalli E, et al. DAX1, a direct target of EWS/FLI1 oncoprotein, is a principal regulator of cell-cycle progression in Ewing's tumor cells. Oncogene. 2008;27:6034-43.

24. Monument MJ, Johnson KM, McIlvaine E, et al. Clinical and Biochemical Function of Polymorphic NR0B1 GGAA-Microsatellites in Ewing Sarcoma: A Report from the Children's Oncology Group. PLoS ONE. 2014;9:e104378.

25. Johnson KM, Taslim C, Saund RS, et al. Identification of two types of GGAA-microsatellites and their roles in EWS/FLI binding and gene regulation in Ewing sarcoma. PLoS ONE. 2017;12: e0186275.

26. Laurini JA, Cooper K, Fletcher CDM, et al. Protocol for the Examination of Specimens From Patients With Soft Tissue Tumors, College of American Pathologists. Version: Soft Tissue 4.0.0.0, http://www.cap.org/ShowProperty?nodePath=/UCMCon/ Contribution $\% 20$ Folders/WebContent/pdf/cp-softtissue17protocol-4000.pdf, accessed 15 April 2018.

27. Fletcher CDM, Bridge JA, Hogendoorn PCW, Mertens F, eds. WHO Classification of Tumours of Soft Tissue and Bone. 4th ed. Geneva, Switzerland: WHO Press; 2013. 